\title{
Processing of Own Hand Visual Feedback during Object Grasping in Ventral Premotor Mirror Neurons
}

\author{
Monica Maranesi, ${ }^{1}$ Alessandro Livi, ${ }^{2}$ and Luca Bonini ${ }^{1}$ \\ ${ }^{1}$ Istituto Italiano di Tecnologia, Brain Center for Social and Motor Cognition, 43125 Parma, Italy, and ${ }^{2}$ Dipartimento di Neuroscienze, Università di Parma, \\ 43125 Parma, Italy
}

\begin{abstract}
Mirror neurons (MNs) discharge during action execution as well as during observation of others' actions. Our own actions are those that we have the opportunity to observe more frequently, but no study thus far to our knowledge has addressed the issue of whether, and to what extent, MNs can code own hand visual feedback (HVF) during object grasping. Here, we show that MNs of the ventral premotor area F5 of macaque monkeys are particularly sensitive to HVF relative to non-MNs simultaneously recorded in the same penetrations. Importantly, the HVF effect is more evident on MN activity during hand-object interaction than during the hand-shaping phase. Furthermore, the increase of MN activity induced by HVF and others' actions observed from a subjective perspective were positively correlated. These findings indicate that at least part of ventral premotor MNs can process the visual information coming from own hand interacting with objects, likely playing a role in self-action monitoring.
\end{abstract}

Key words: action observation; area F5; visuomotor processing

\section{Significance Statement}

We show that mirror neurons (MNs) of area F5 of the macaque, in addition to encoding others' observed actions, are particularly sensitive, relative to simultaneously recorded non-MNs, to the sight of the monkey's own hand during object grasping, likely playing a role in self-action monitoring.

\section{Introduction}

Mirror neurons (MNs) have the intriguing property of firing not only during the execution of an action but also when one observes a similar action performed by another individual (Rizzolatti et al., 2014). Recent evidence indicates that the majority of MNs in the monkey premotor area F5 respond, sometimes even selectively, to the observation of filmed actions viewed from a subjective perspective (Caggiano et al., 2011). Interestingly, the same investigators showed that observing an action from a first-person perspective produce local field potentials more similar to those generated by action execution (in the dark) than by the observation of the action from a frontal view (Caggiano et al., 2015). Indeed, our own actions are those that we have the opportunity to observe more frequently, but whether and to what extent the

Received Jan. 23, 2015; revised July 13, 2015; accepted July 20, 2015.

Author contributions: M.M. and L.B. designed research; M.M., A.L., and L.B. performed research; M.M., A.L., and L.B. analyzed data; M.M. and L.B. wrote the paper.

This work was supported by the Istituto Italiano di Tecnologia and the European Commission Grant Cogsystem (Grant FP7-250013). We thank Corrado Sinigaglia and Fausto Caruana for their helpful comments on an early version of the manuscript.

Correspondence should be addressed to either Monica Maranesi, PhD, or Luca Bonini, PhD, Istituto Italiano di Tecnologia, Brain Center for Social and Motor Cognition, Via Volturno 39, 43125 Parma, Italy; E-mail: monica.maranesi@iit.it or luca.bonini@unipr.it.

DOI:10.1523/JNEUROSCI.0301-15.2015

Copyright $\odot 2015$ the authors $\quad 0270-6474 / 15 / 3511824-06 \$ 15.00 / 0$ sight of one's own hand during grasping affects MN motor discharge remains unknown.

Here, we addressed this issue by studying MN activity while monkeys grasped an object with and without hand visual feedback (HVF) and when they observed (from a "first-person" perspective) an experimenter grasping.

\section{Materials and Methods}

\section{Subjects}

Experiments were performed on two male adult monkeys: Mk1 (Macaca nemestrina, $9 \mathrm{~kg}$ ) and Mk2 (Macaca mulatta, $7 \mathrm{~kg}$ ). Before recordings, monkeys were trained to perform the task described below with the hand (left) contralateral to the hemisphere to be recorded (right). A head fixation system and a recording chamber were implanted under general anesthesia (ketamine hydrocloride, $5 \mathrm{mg} / \mathrm{kg}$, i.m., and medetomidine hydrocloride, $0.1 \mathrm{mg} / \mathrm{kg}$, i.m.), followed by postsurgical pain medications, as described previously (Bonini et al., 2012). All experiments were performed in accordance with protocols approved by the Veterinarian Animal Care and Use Committee of the University of Parma and with the European law on the humane care and use of laboratory animals.

\section{Recorded region and behavioral paradigm}

Recordings were performed from the hand region of the ventral premotor area F5 (Fig. 1A) (see Bonini et al., 2014b for detailed functional mapping of the investigated sector). Monkeys were trained to perform a visuomotor task (execution) and, in a subsequent block of trials, to ob- 
A

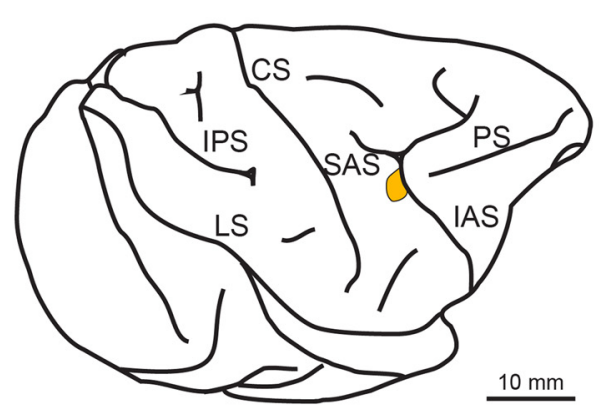

B

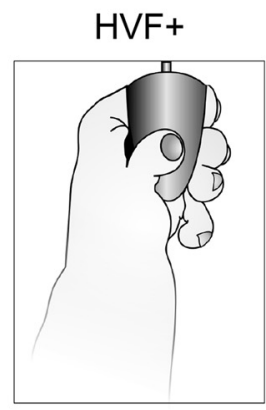

HVF-

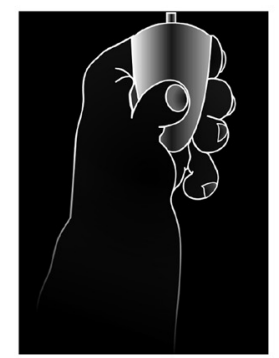

OBS

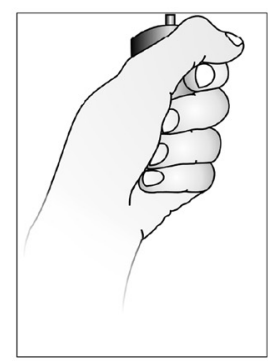

C

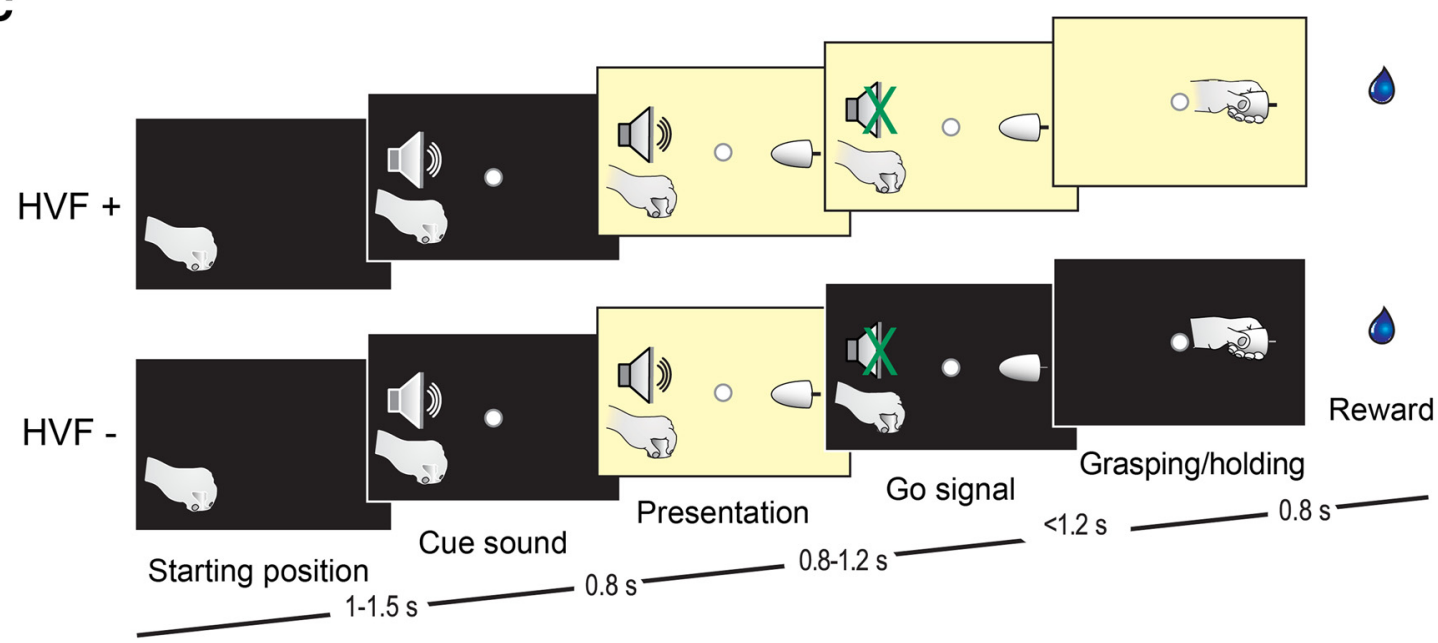

Figure 1. A, Highlighted sector denotes the portion of the ventral premotor area $\mathrm{F} 5$ from which neurons were recorded. IPS, Intraparietal sulcus; $\mathrm{CS}$, central sulcus; $\mathrm{SAS}$, spur of the arcuate sulcus; IAS, inferior arcuate sulcus; $\mathrm{PS}$, principal sulcus. $B$, Schematic representation of the three main tested conditions: grasping with $\left(\mathrm{HVF}^{+}\right.$) and without ( $\mathrm{HVF}^{-}$) hand visual feedback and observation of the experimenter's grasping from a first-person perspective (OBS). C, Schematic representation of the temporal structure of the task events. The sequence of events of $\mathrm{HVF}^{+}{ }^{+}$and $\mathrm{OBS}$ condition was the same.

serve the experimenter (from a first-person visual perspective) performing the same task in their stead (observation).

The apparatus used for the task has been described in detail previously (Bonini et al., 2014b). The execution task included two basic conditions (Fig. 1B): (1) grasping in the light (i.e., with hand visual feedback, $\mathrm{HVF}^{+}$) and (2) grasping in the dark (i.e., without hand visual feedback, HVF-). The observation task (Fig. $1 B$ ) required the monkey to remain still with its hand on the starting position while observing the experimenter grasping the object. Although the animal grasped three different objects during the execution task (Bonini et al., 2014b), only one object (a big metallic cone, base diameter $30 \mathrm{~mm}$, length $45 \mathrm{~mm}$ ) was used for testing action observation from a subjective perspective: therefore, we focused on this same object for the analysis of motor responses in the two conditions.

Execution task. For the execution task (Fig. 1C), a fixation point was presented in the exact position of the (not yet visible) target object in complete darkness and the monkey was required to start fixating it within $1.2 \mathrm{~s}$. Fixation onset determined the presentation of a cue sound (a 1200 $\mathrm{Hz}$ sine wave). After $0.8 \mathrm{~s}$, the target object became visible. After a variable time lag $(0.8-1.2 \mathrm{~s})$, the sound ceased (go signal). At this point during $\mathrm{HVF}^{+}$, the light remained switched on until the end of the trial, whereas during $\mathrm{HVF}^{-}$, the light switched off simultaneously with the go signal and the monkey reached, grasped, and pulled (for at least $0.8 \mathrm{~s}$ ) the object in the light $\left(\mathrm{HVF}^{+}\right)$or in the dark $\left(\mathrm{HVF}^{-}\right)$, respectively. The two conditions were randomly interleaved, thus ensuring that the monkey could not predict in advance of the go signal whether it had to grasp in the light or in the dark. The fixation point remained visible for the entire duration of the task, serving as a visual guidance, particularly for reaching in the dark.
Observation task. For the observation task, the temporal sequence of sensory events was the same as the execution task, but the monkey remained still and maintained fixation while observing the experimenter performing the action. The experimenter's (left) hand starting position was located $10 \mathrm{~cm}$ to the left of the monkey's left hand. LabView-based software monitored the tasks stages: if the monkey broke fixation, made an incorrect movement, or did not respect the task's temporal constraints, the trial was aborted and no reward was delivered. After the monkey correctly performed trials, a fixed amount of juice was delivered automatically.

\section{Recording techniques}

Neuronal recordings were performed by means of 16 channels multielectrode linear arrays: U-probes (Plexon,) and silicon probes developed in the EU project NeuroProbes (Herwik et al., 2011) and distributed by ATLAS Neuroengineering (Belgium). Details on the devices and techniques used to handle the probes have been provided previously (Bonini et al., 2014a, 2014b). The signal was amplified and sampled at $40 \mathrm{kHz}$ with a 16-channel Omniplex system (Plexon). Online spike sorting was performed with dedicated software (Plexon), but all final quantitative analyses were performed offline.

Recording of behavioral events and definition of epochs of interest Contact-sensitive devices (Crist Instruments) were used to detect when the monkey or the experimenter touched the metal surface of the starting position or the target object. A switch located behind the object signaled the onset of object pulling. Each of these devices provided a TTL signal that is used by LabView-based software to monitor and control the task unfolding and is recorded in parallel with neuronal activity, enabling subsequent alignment of neuron response and statistical analyses. 
A
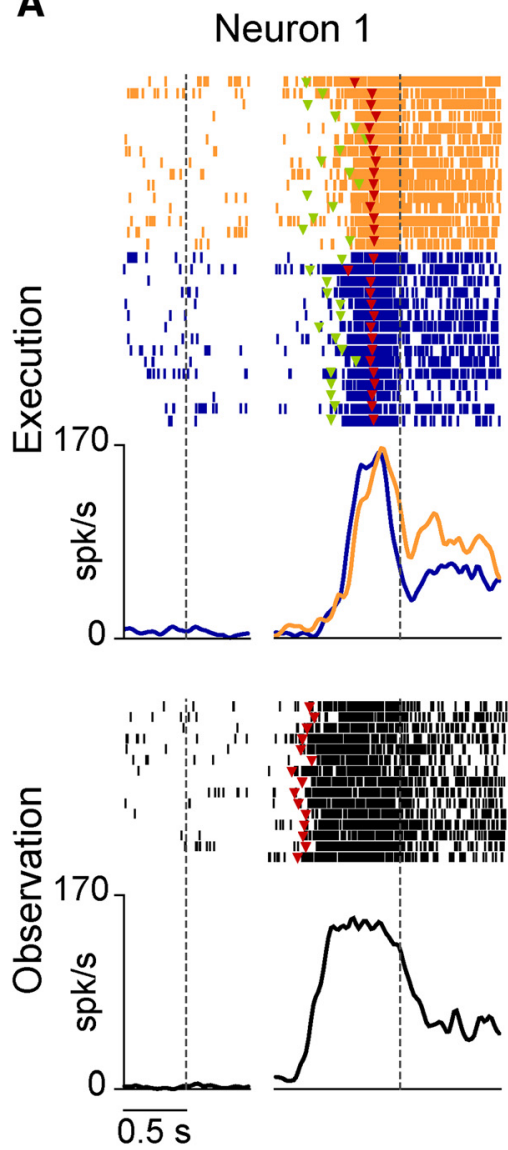

B

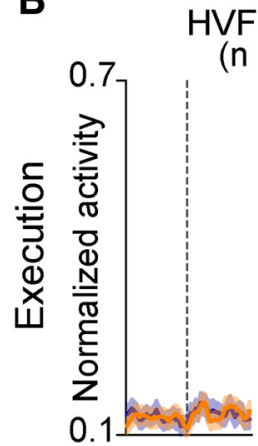

$\mathrm{HVF}+\mathrm{MNs}$
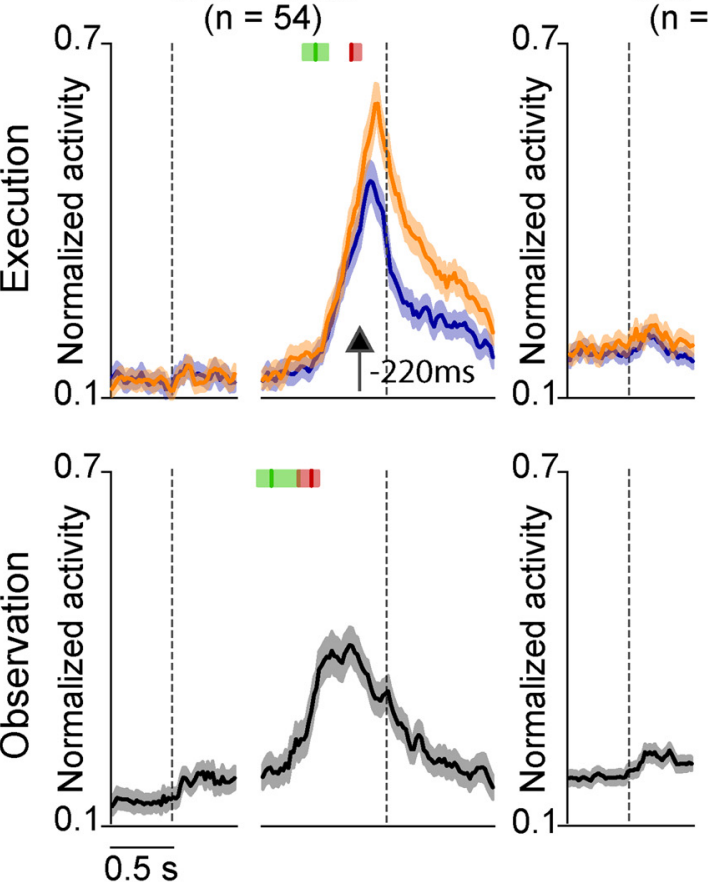

Neuron 2
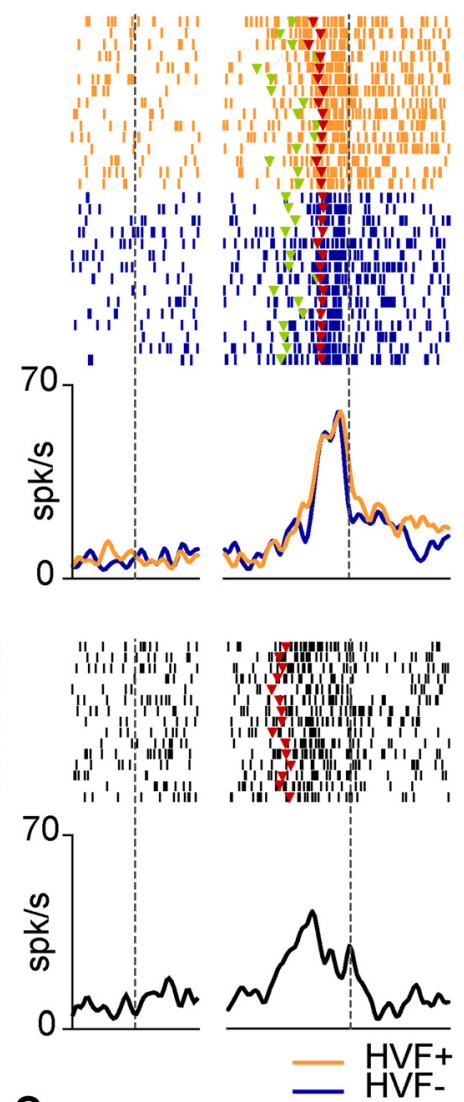

C
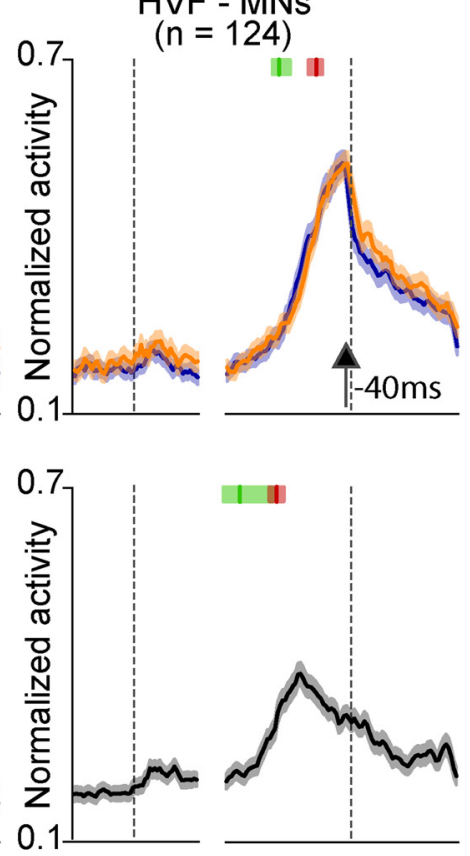

Figure 2. $\quad \boldsymbol{A}$, Each panel shows the raster plot (top) and peristimulus spike density function (bottom) of two MNs aligned on object presentation (first dashed line) and then (after the gap) on the onset of object pulling performed by the monkey (execution, $\mathrm{HVF}^{+}$: orange; $\mathrm{HVF}^{-}$: blue) or experimenter (observation: gray). Note that most of the differential activity in the HVF ${ }^{+} \mathrm{MN}$ occurs during the static hand- object interaction epoch (holding) rather than during the hand-shaping epoch. Colored triangular markers represent the go signal (green) and the detachment of the hand from the starting position (red). $\boldsymbol{B}, \boldsymbol{C}$, Population responses of $\mathrm{HVF}^{+}$and HVF ${ }^{-}$MNs aligned as the neuron examples in $\boldsymbol{A}$. Shaded regions represent $1 \mathrm{SEM}$. The median time of the go signal and reaching onset are indicated with the green and red markers, respectively: the shaded areas around each marker represent the $25^{\text {th }}$

Eye position was monitored with an eye tracking system (pupil) composed of a $50 \mathrm{~Hz}$ infrared-sensitive CCD video camera and two spots of infrared light. The monkey was required to maintain its gaze on the fixation point (tolerance radius $5^{\circ}$ ) during all task phases.

Different epochs of interest were defined: (1) baseline, $500 \mathrm{~ms}$ before object presentation; (2) object presentation, from 50 to $450 \mathrm{~ms}$ after switching on the light; (3) hand shaping, from the detachment of the hand from the starting position to the beginning of object pulling (note that this epoch is subject to trial-by-trial variability, enabling us to capture specifically the hand movement phase in each trial); and (4) object holding, from pulling onset to 500 ms after this event. The same epochs were used to analyze neuronal responses during both grasping execution and observation.

We recorded the EMG activity in both monkeys by placing couples of surface $\mathrm{Ag}-\mathrm{AgCl}$ electrodes over two muscles, the left extensor digitorum communis and the deltoid. Data were band-pass filtered between 30 and $500 \mathrm{~Hz}$ (fourth-order Butterworth), rectified, and averaged across trials for plotting. The muscle activation was analyzed with $2 \times 2$ repeatedmeasures ANOVAs (factors: condition and epoch, with a significance criterion of $p<0.01$ ) for each muscle separately.

\section{Single neuron analyses}

Single units were isolated using principal component and template matching techniques provided by dedicate offline sorting software (Plexon), as described previously (Bonini et al., 2014b).

Preliminary analyses of the recorded data were performed to identify grasping neurons with mirror properties. To identify grasping neurons, we applied a one-way repeatedmeasures ANOVA (4 levels of the factor epoch, with a significance criterion of $p<0.01$ ) to each neuron response in $\mathrm{HVF}^{+}$and $\mathrm{HVF}^{-}$conditions of the execution task separately: the activity had to be significantly stronger during the hand-shaping and/or object-holding epoch relative to both baseline and object presentation epochs $(p<$ 0.05 , Bonferroni corrected). To identify $\mathrm{MNs}$, we analyzed the activity of grasping neurons during the observation task: those showing stronger discharge during hand shaping and/or object holding relative to both baseline and object presentation epochs of the observation task were classified as MNs. Each neuron was considered to be modulated in a specific condition if it reached a firing rate of at least 5 spikes/s in at least 1 epoch of that condition. Neurons with

and $75^{\text {th }}$ percentile times of other events of the same type. Black arrows indicate the time of the first of a series of at least five consecutive $100 \mathrm{~ms}$ bins in which population activity differed significantly between the two conditions (pairedsamples $t$ tests, $p<0.05$ ) 
A

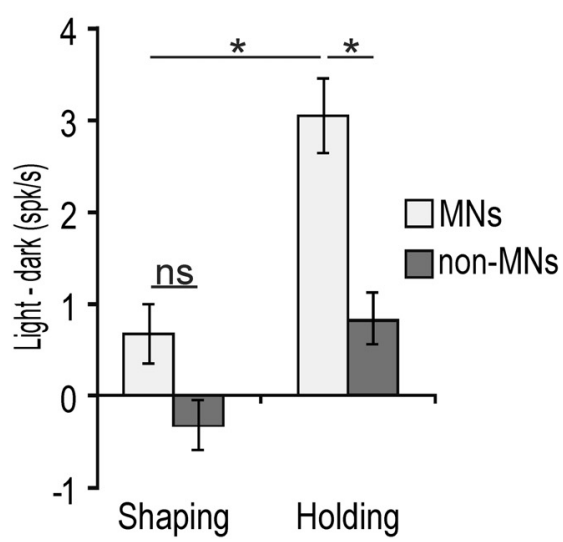

Figure 3. A, Differential activity between $\mathrm{HVF}^{+}$and $\mathrm{HVF}^{-}$conditions during shaping and holding epochs in all the recorded neurons $\left(\mathrm{HVF}^{+}\right.$and $\mathrm{HVF}^{-}$) with (MNs) and without (non-MNs) mirror properties. ${ }^{*} p<0.001$. B, Correlation plot between HVF and grasping observation activity in $\mathrm{HVF}^{+} \mathrm{MNs}$ (see Materials and Methods). The dashed line represents the function $x=y$. Note that, by performing the same analysis after removal of pairs of entries in which one or both of the values exceeded 2 SDs from the mean of their distribution $(n=3)$, the correlation remained significant $(r=0.4, p=0.0013)$.

inhibitory responses are not included in the present dataset because they represented $<4 \%$ of all recorded neurons. Single neuron sensitivity to HVF was assessed by comparing their response during hand shaping and object holding epochs of $\mathrm{HVF}^{+}$and $\mathrm{HVF}^{-}$conditions by means of a $2 \times 2$ repeated-measures ANOVA (factors: condition and epoch, with a significance criterion of $p<0.01$ for either condition or condition $\times$ epoch effects). Neurons were recorded for 15 correctly performed trials in each condition.

Possible correlation between the modulation induced by HVF (HVF effect) and that associated with other's observed action was assessed as follows: the HVF effect was computed by subtracting the activity during grasping in $\mathrm{HVF}^{-}$condition to the one of the corresponding epoch/s of the $\mathrm{HVF}^{+}$condition based on the results of single neuron analysis. To estimate the activity induced by other's observed action, we subtracted the neuron response during the object presentation epoch from that during grasping observation in the observation task.

\section{Population analyses}

Neuronal activity was first normalized to the highest firing rate of each neuron by taking into account both the execution and observation tasks and then averaged across trials to obtain a population vector for each neuron in each condition. The population response was computed by averaging activity across neurons and analyzed with $2 \times 2$ repeatedmeasures ANOVAs (factors: condition and epoch), followed by Bonferroni post hoc tests. Because trial-by-trial data could not be used in this case, we considered the following epochs: (1) hand shaping, the epoch corresponding to $500 \mathrm{~ms}$ before pulling onset, and (2) object holding, the $500 \mathrm{~ms}$ epoch after the same event.

\section{Results}

We recorded 376 grasping neurons from the ventral premotor area F5 of the two monkeys. Almost half of them $(n=181$, $48.1 \%$ ) showed mirror properties (MNs), whereas the remaining did not respond during action observation (non-MNs, $n=195$, $51.9 \%$ ). Only $3.5 \%$ of the recorded neurons discharged stronger during grasping in the dark than in the light ( $n=3 \mathrm{MNs}, n=10$ non-MNs): due to their low number, these cells were not considered in the subsequent analyses. Importantly, a higher proportion of MNs $(54 / 178,30.3 \%)$ than non-MNs $\left(21 / 185,11.4 \% \chi^{2}\right.$ $=19.95, p<0.001)$ discharged more strongly when the HVF was available.

Figure $2 A$ shows examples of the recorded MNs. Neuron 1 discharged stronger during the $\mathrm{HVF}^{+}$than the $\mathrm{HVF}^{-}$condition, thus being influenced by monkey's hand visual feedback $\left(\mathrm{HVF}^{+}\right.$
$\mathrm{MN})$. In contrast, neuron 2 discharged similarly $(p>0.05)$ in the two conditions, thus showing no sensitivity to the hand visual feedback ( $\left.\mathrm{HVF}^{-} \mathrm{MN}\right)$. The majority of $\mathrm{HVF}^{+}$MNs showed the HVF effect during hand-object interaction: $76 \%$ were significantly modulated during both the hand-shaping and object-holding epochs, $17 \%$ during the holding epoch only, and the remaining $7 \%$ showed an HVF effect selectively during hand shaping. Population analyses showed that the HVF effect started $220 \mathrm{~ms}$ before hand-target contact, but it characterized the whole object-holding epoch in $\mathrm{HVF}^{+} \mathrm{MNs}$ (Fig. $2 B)$. Interestingly, although not significant when assessed at the single neuron level, a significant HVF effect emerged also among $\mathrm{HVF}^{-} \mathrm{MNs}$ when considered at the population level (interaction condition $\times$ epoch: $\left.F_{(1,123)}=28.14, p<0.001\right)$, but only during the object-holding epoch (Bonferroni post hoc $p<0.001$; Fig. 2C). This did not occur among $\mathrm{HVF}^{-}$non-MNs despite their high number $(n=164)$, suggesting that the HVF influences more specifically the response of the neurons with mirror properties.

To better scrutinize this effect, we performed a $2 \times 2$ repeatedmeasures ANOVA on the light-dark differential activity during the hand-shaping and object-holding epochs, including neuronal category (MNs and non-MNs) as an additional grouping factor. The results showed significant main effects for both factors $(p<0.001)$, as well as a significant interaction effect $\left(F_{(1,361)}=4.63, p=0.03\right)$ : Bonferroni post hoc tests indicated that MNs are more affected by the HVF than non-MNs $(p<0.001)$ during the hand-object interaction (holding) epoch (Fig. $3 A$ ). In addition, the modulations induced by the HVF and other's observed action on $\mathrm{HVF}^{+} \mathrm{MN}$ activity were positively and significantly correlated $(r=0.74, p<$ 0.001 ), suggesting that these neurons process in a similar way the visual information on self and others' action (Fig. 3B).

\section{Control behavioral data}

Both monkey (Fadiga et al., 2013) and human (Winges et al., 2003) studies suggest that grasping in the dark can cause an increase of the reach-to-grasp time relative to grasping in the light. However, this does not affect hand shaping (Winges et al., 2003), particularly when the availability of visual feedback is unpredictable until movement onset (Jakobson and Goodale, 1991), as in the case of our task. Indeed, we found that the reaching time did not differ between $\mathrm{HVF}^{+}$and $\mathrm{HVF}^{-}$conditions in either monkey (Fig. 4A).

In further control experiments, we recorded EMG activity during $\mathrm{HVF}^{+}$and $\mathrm{HVF}^{-}$conditions, as well as during grasping observation (Fig. 4B). It is clear that, for both the proximal (deltoid) and distal (extensor digitorum communis) recorded limb muscles, no modulation occurred during action observation, which is consistent with previous MN studies with different paradigms (Kraskov et al., 2009). Furthermore, no significant difference was observed between muscle activity during $\mathrm{HVF}^{+}$and $\mathrm{HVF}^{-}$conditions in either monkey, thus supporting the idea that the modulations of neuronal activity reflect the processing of own hand visual feedback during object grasping. 
A

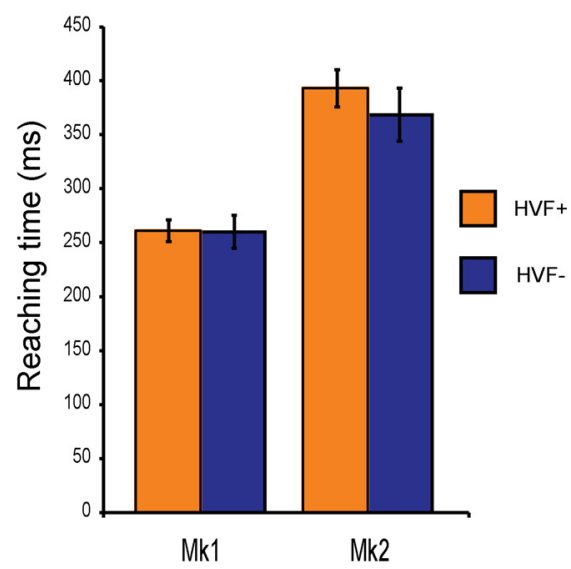

B
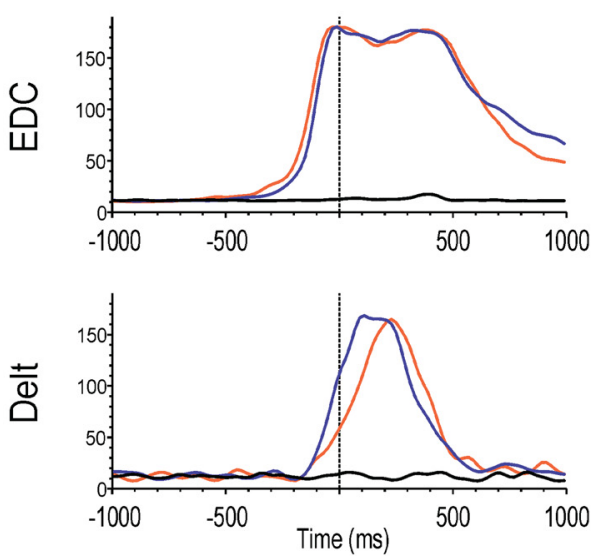

Mk2
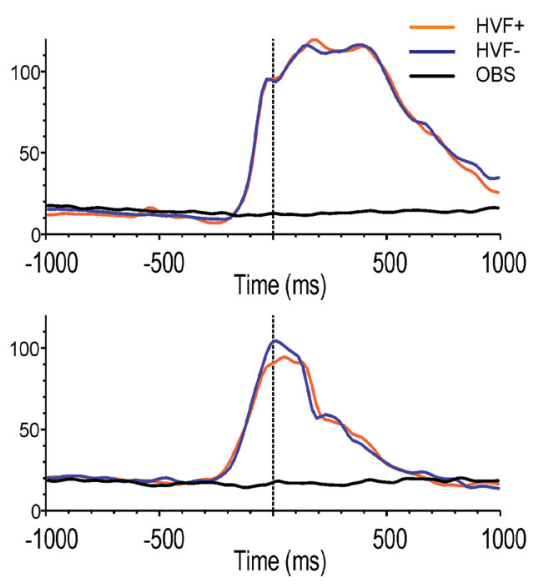

Figure 4. $\quad A$, Reaching time during grasping in $\mathrm{HVF}^{+}$and $\mathrm{HVF}^{-}$conditions. Although the two animals performed the action at different speed, there is no significant difference between the two

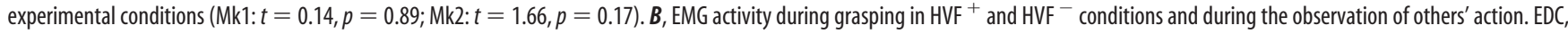
Extensor digitorum communis; Delt, deltoid.

\section{Discussion}

The main goal of this study was to verify whether, and to what extent, the motor discharge of area F5 MNs, which typically fire during the observation of others' action, also encodes the visual feedback of monkey's own hand during action execution. Previous studies described neurons influenced by HVF in the ventral premotor (Raos et al., 2006; Fadiga et al., 2013), dorsal premotor (Raos et al., 2004), and posterior parietal (Sakata et al., 1995; Battaglia-Mayer et al., 2001; Bosco et al., 2010; Maeda et al., 2015) cortex, but nothing was known concerning MNs, fueling the original idea (Gallese et al., 1996) that these cells are specifically sensitive to the sight of others' action.

Here, we report that the motor discharge of $\sim 30 \%$ of F5 MNs is influenced by HVF. However, alternative interpretations of this finding need to be considered. For example, the HVF effect could have been caused by possible differences in the monkeys' performance of the task during light and dark conditions. To avoid this problem, we designed the task on the basis of previous studies (Winges et al., 2003) to render the visibility of both the hand and the target object not necessary for action execution. In particular, monkeys were overtrained in performing the task, the availability of HVF was unpredictable until the go signal, and the fixation point remained visible on the center of the object during the whole trial to provide a visual guidance during grasping in the dark. Indeed, the similarity of reaching times and EMG activation profiles recorded during $\mathrm{HVF}^{+}$and $\mathrm{HVF}^{-}$conditions in both monkeys suggest that the lack of HVF does not alter the monkeys' performance and thus cannot account for the HVF effect on neuron discharge. Another possible interpretation is that the HVF effect depends on the sight of the target object. However, even this interpretation is unlikely because virtually all $(48 / 54) \mathrm{HVF}^{+}$ MNs failed to show any significant activation to the visual presentation of the target. Furthermore, recent single neuron studies on inferior parietal lobule, a crucial source of visual information for area F5 (Rizzolatti et al., 2014), used videos of actions in which the presence/absence of the target object could be accurately manipulated (Pani et al., 2014; Maeda et al., 2015) and showed that neurons with mirror-like properties discharged similarly during action observation regardless of the presence/absence of the target object. Our finding that the HVF influences F5 MN activity during hand-object interaction (when the monkey's hand hides the object), together with the previous data, suggest that the sight of the grasping hand, rather than of the object or the hand visual image by themselves, is the crucial factor that modulates F5 MN activity.

We also showed that MNs are particularly sensitive to HVF relative to non-MNs, although both of these types of cells do activate during grasping execution. In addition, even those MNs that did not show a significant HVF effect when analyzed individually did show a significant modulation at the population level. This effect was specific for MNs because it did not occur in grasping neurons lacking mirror properties, despite their even higher number, and it specifically occurred during the handobject interaction phase (Fig. $3 A$ ). These findings may appear to be in contrast with recent data in monkeys (Fadiga et al., 2013) showing that the discharge of more than half of F5 non-MNs exhibited light/dark modulation. However, in that study, the investigators very likely explored mainly those sectors of area F5 devoid of visual responses (Maranesi et al., 2012), whereas mirror and non-MNs in our study were recorded simultaneously during the same penetrations. Indeed, Fadiga et al. (2013) found that the majority (64\%) of light/dark-modulated neurons discharged stronger in the dark, whereas this type of neuron was virtually absent $(3.5 \%)$ in our dataset. Therefore, the HVF modulation is very likely the result of different mechanisms and plays different roles depending on the cortical region in which it occurs.

Why does HVF affect MNs more specifically than non-MNs? Based on most ontogenetic hypotheses on MN origin (Del Giudice et al., 2009; Casile et al., 2011; Oztop et al., 2013), all of the cells destined to become MNs are originally sensitive to the sight of infant's own actions and experience-based generalization processes would then endow neuronal responses with different degree of selectivity for self- or others' observed actions. Therefore, the visual feedback from own hand during grasping might have retained a particular relevance for MNs. Indeed, we found significant correlations between the magnitude of the modulation induced by the HVF and that caused by others' observed action in $\mathrm{HVF}^{+} \mathrm{MNs}$ (Fig. 3B), supporting the idea that this particular set of MNs can process in a similar way visual information on both self and others' action. The existence of neuronal populations with selective responses for self or others' action has also been documented in both mesial premotor (Yoshida et al., 2011) and 
inferior parietal (Maeda et al., 2015) cortex, which are anatomically linked to area F5 (Rozzi et al., 2006; Borra et al., 2008; Gerbella et al., 2011), suggesting that a wide system of interconnected areas is involved in the processing of HVF and others' observed actions.

MN sensitivity to own hand visual feedback during object grasping was predicted by previous computational models (Bonaiuto and Arbib, 2010) suggesting that a possible additional function of the MN system consists of self-action monitoring. If one accepts this view, then $\mathrm{HVF}^{+} \mathrm{MNs}$ can represent a valuable resource of neural plasticity for the fine tuning of $\mathrm{MN}$ properties during sensorimotor learning (Wiggett et al., 2012; Oztop et al., 2013).

\section{References}

Battaglia-Mayer A, Ferraina S, Genovesio A, Marconi B, Squatrito S, Molinari M, Lacquaniti F, Caminiti R (2001) Eye-hand coordination during reaching. II. An analysis of the relationships between visuomanual signals in parietal cortex and parieto-frontal association projections. Cereb Cortex 11:528-544. CrossRef Medline

Bonaiuto J, Arbib MA (2010) Extending the mirror neuron system model, II: what did I just do? A new role for mirror neurons. Biol Cybern 102: 341-359. CrossRef Medline

Bonini L, Ugolotti Serventi F, Bruni S, Maranesi M, Bimbi M, Simone L, Rozzi S, Ferrari PF, Fogassi L (2012) Selectivity for grip type and action goal in macaque inferior parietal and ventral premotor grasping neurons. J Neurophysiol 108:1607-1619. CrossRef Medline

Bonini L, Maranesi M, Livi A, Bruni S, Fogassi L, Holzammer T, Paul O, Ruther P (2014a) Application of floating silicon-based linear multielectrode arrays for acute recording of single neuron activity in awake behaving monkeys. Biomed Tech (Berl) 59:273-281. Medline

Bonini L, Maranesi M, Livi A, Fogassi L, Rizzolatti G (2014b) Spacedependent representation of objects and other's action in monkey ventral premotor grasping neurons. J Neurosci 34:4108-4119. CrossRef Medline

Borra E, Belmalih A, Calzavara R, Gerbella M, Murata A, Rozzi S, Luppino G (2008) Cortical connections of the macaque anterior intraparietal (AIP) area. Cereb Cortex 18:1094-1111. CrossRef Medline

Bosco A, Breveglieri R, Chinellato E, Galletti C, Fattori P (2010) Reaching activity in the medial posterior parietal cortex of monkeys is modulated by visual feedback. J Neurosci 30:14773-14785. CrossRef Medline

Caggiano V, Fogassi L, Rizzolatti G, Pomper JK, Thier P, Giese MA, Casile A (2011) View-based encoding of actions in mirror neurons of area $\mathrm{f5}$ in macaque premotor cortex. Curr Biol 21:144-148. CrossRef Medline

Caggiano V, Giese M, Thier P, Casile A (2015) Encoding of point of view during action observation in the local field potentials of macaque area F5. Eur J Neurosci 41:466-476. Medline

Casile A, Caggiano V, Ferrari PF (2011) (2011) The mirror neuron system: a fresh view. Neuroscientist 17:524-538. CrossRef Medline

Del Giudice M, Manera V, Keysers C (2009) Programmed to learn? The ontogeny of mirror neurons. Dev Sci 12:350-363. CrossRef Medline

Fadiga L, Caselli L, Craighero L, Gesierich B, Oliynyk A, Tia B, Viaro R (2013) Activity in ventral premotor cortex is modulated by vision of own hand in action. Peer J 1:e88. CrossRef Medline
Gallese V, Fadiga L, Fogassi L, Rizzolatti G (1996) Action recognition in the premotor cortex. Brain 119:593-609. Medline

Gerbella M, Belmalih A, Borra E, Rozzi S, Luppino G (2011) Cortical connections of the anterior (F5a) subdivision of the macaque ventral premotor area F5. Brain Struct Funct 216:43-65. CrossRef Medline

Herwik S, Paul O, Ruther P (2011) Ultrathin silicon chips of arbitrary shape by etching before grinding. Journal of Microelectromechanical Systems 20:791-793. CrossRef

Jakobson LS, Goodale MA (1991) Factors affecting higher-order movement planning: a kinematic analysis of human prehension. Exp Brain Res 86: 199-208. Medline

Kraskov A, Dancause N, Quallo MM, Shepherd S, Lemon RN (2009) Corticospinal neurons in macaque ventral premotor cortex with mirror properties: a potential mechanism for action suppression? Neuron 64: 922-930. CrossRef Medline

Maeda K, Ishida H, Nakajima K, Inase M, Murata A (2015) Functional properties of parietal hand manipulation-related neurons and mirror neurons responding to vision of own hand action. J Cogn Neurosci 27: 560-572. CrossRef Medline

Maranesi M, Rodà F, Bonini L, Rozzi S, Ferrari PF, Fogassi L, Coudé G (2012) Anatomo-functional organization of the ventral primary motor and premotor cortex in the macaque monkey. Eur J Neurosci 36:3376-3387. CrossRef Medline

Oztop E, Kawato M, Arbib MA (2013) Mirror neurons: functions, mechanisms and models. Neurosci Lett 540:43-55. CrossRef Medline

Pani P, Theys T, Romero MC, Janssen P (2014) Grasping execution and grasping observation activity of single neurons in the macaque anterior intraparietal area. J Cogn Neurosci 26:2342-2355. CrossRef Medline

Raos V, Umiltá MA, Gallese V, Fogassi L (2004) Functional properties of grasping-related neurons in the dorsal premotor area $\mathrm{F} 2$ of the macaque monkey. J Neurophysiol 92:1990-2002. CrossRef Medline

Raos V, Umiltá MA, Murata A, Fogassi L, Gallese V (2006) Functional properties of grasping-related neurons in the ventral premotor area F5 of the macaque monkey. J Neurophysiol 95:709-729. Medline

Rizzolatti G, Cattaneo L, Fabbri-Destro M, Rozzi S (2014) Cortical mechanisms underlying the organization of goal-directed actions and mirror neuron-based action understanding. Physiol Rev 94:655-706. CrossRef Medline

Rozzi S, Calzavara R, Belmalih A, Borra E, Gregoriou GG, Matelli M, Luppino G (2006) Cortical connections of the inferior parietal cortical convexity of the macaque monkey. Cereb Cortex 16:1389-1417. Medline

Sakata H, Taira M, Murata A, Mine S (1995) Neural mechanisms of visual guidance of hand action in the parietal cortex of the monkey. Cereb Cortex 5:429-438. CrossRef Medline

Wiggett AJ, Hudson M, Clifford A, Tipper SP, Downing PE (2012) Doing, seeing, or both: effects of learning condition on subsequent action perception. Soc Neurosci 7:606-621. CrossRef Medline

Winges SA, Weber DJ, Santello M (2003) The role of vision on hand preshaping during reach to grasp. Exp Brain Res 152:489-498. CrossRef Medline

Yoshida K, Saito N, Iriki A, Isoda M (2011) Representation of others' action by neurons in monkey medial frontal cortex. Curr Biol 21:249-253. Medline 